\title{
Detection Criterion on Airborne Sensor Measure Information Effectiveness
}

\author{
Bo Liu ${ }^{1}$, Weijia Wang ${ }^{2}$, Bingsong Xiao ${ }^{2, a, *}$, Zhiying Peng ${ }^{2}$ \\ ${ }^{1}$ Science and Technology on Avionics Integration Laboratory, Shanghai 200233, China \\ ${ }^{2}$ Aeronautics and Astronautics Engineering College, Air Force Engineering University, Xi'an \\ 710038, China \\ ${ }^{a}$ Corresponding author: Bingsong Xiao. email: wang_mn@163.com
}

Keywords: Sensor, Incomplete measurement, error covariance Cramer-Rao bound,

Abstract: Based on the detection criterion of traditional single threshold residual information, in order to get likelihood probability, this paper makes residual information fuzzy which is in the range of the upper and lower bounds (UBL for short) of sensor residual information effectiveness that is under incomplete measurement respectively set by steady-state error covariance under incomplete measurement and posterior Cramer-Rao bound (CRB). It advances the detection criteria on the basis of UBL of residual under incomplete measurement on the premise of residual information confidence at the moment according to Bayes formula and accomplishes the simulation.

\section{Introduction}

Imprecision, inaccuracy and vagueness included in sensor measure information jointly lead to uncertainty, which makes the assessment on sensor measure information effectiveness especially important under incomplete measurement ${ }^{[1]}$. Effectiveness of measurement data is commonly explained as the error between measured value and true value on targets, namely the smaller error is, the higher the effectiveness will be ${ }^{[2]}$. Hadidi and Schwartz ${ }^{[3]}$ combine incomplete measurement sequence and Markov sequence utilizing the same distribution but not absolute with other random variable to describe incomplete measure, and offer the corresponding filter equation. This paper points at analyzing single sensor measure residual information and combines outliers' judge rule proposing basing upon effectiveness limitation residual detection norm.

\section{UBL of residual effectiveness under incomplete measurement}

\subsection{Upper bound of residual effectiveness}

To Analyses filtered process that target is steadily tracked when the detection probability of sensors is constant. Considering that is under incomplete measurement, state equation and measurement equation of target tracking process at the time of $k$ :

$$
\begin{aligned}
& X_{k+1}=A_{k} X_{k}+w_{k} \\
& y_{k+1}=d_{k} C_{k} X_{k}+v_{k}
\end{aligned}
$$

According to the error variance value that when tracking system reach stability level under the 
condition that the target environment is constant can be get:

$$
P=(1-\lambda)\left(A P A^{T}+Q\right)+\lambda\left[(I-K C)\left(A P A^{T}+Q\right)(I-K C)^{T}+K R K^{T}\right]
$$

Considering the relationship between filtering residual error covariance and estimate error covariance which is:

$$
E\left[v(k+1) v^{\prime}(k+1)\right]=H(k+1) P H^{T}(k+1)+R(k+1)
$$

So, each component of observed value $Z(k+1)$ at this moment can be distinguished by using $v(k+1)$, it's discriminant formula can be expressed as:

$$
v_{i}(k+1) \leq \sigma_{(i, i)}=a \sqrt{\left[H(k+1) P(k+1 \mid k) H^{\prime}(k+1)+R(k+1)\right]_{i, i}}
$$

In the formula (5): $v_{i}(k+1)$ represents the ith components of $v(k+1) .(i, i)$ represents diagonal elements on line $i$ in the matrix, $a$ is a constant and usually equals to 3 or 4 .

Thus the upper bound of information effectiveness under incomplete measurement is:

$$
\sigma_{(i, i) \max }=a \sqrt{\left[H(k+1) P(k+1 \mid k) H^{\prime}(k+1)+R(k+1)\right]_{i, i}}
$$

If the component satisfies formula (6), the ith component of observation vector $Z(k+1)$ is effective value, otherwise, it is considered as outliers.

Through the analysis of previous section, the upper bound of sensor residual effectiveness is affected by sensor detection probability, noise and other factors under incomplete measurement.

\subsection{Lower bound of residual effectiveness}

The selection of the lower bound of residual information effectiveness is based on CRB of residual estimate in tracking procedure. Literature [4] provides the CRLB of $S_{k}^{l}$ sequence, and the lower bound of effectiveness under incomplete measurement can be get with enumeration method as follow:

$$
J_{k, E N U M}=\sum_{i=1}^{2^{k}} J_{k}^{l} \cdot p\left(S_{k}^{l}\right)=\sum_{i=1}^{2^{k}}\left\{\left(G Q G^{T}+A\left(J_{k-1}^{-1}\right)^{-1} A^{T}\right)^{-1}+d_{i}^{l} H^{T} R^{-1} H\right\} \cdot \prod_{i=1}^{k}\left[d_{i}^{l} \lambda_{i}+\left(1-d_{i}^{l}\right)\left(1-\lambda_{i}\right)\right]
$$

For convenience, literature [5] provides relatively simple CRLB computing methods:

$$
\begin{gathered}
J_{k}^{l}=\lambda H^{T} R^{-1} H+E\left[\left(G Q G^{T}+A\left(J_{k-1}^{l}\right)^{-1} A^{T}\right)^{-1}\right] \leq \lambda H^{T} R^{-1} H+G Q G^{T}+\left\{A\left[E\left(J_{k-1}^{l}\right)\right]^{-1} A^{T}\right\}^{-1} \\
\leq \lambda H^{T} R^{-1} H+G Q G^{T}+\left\{A\left[J_{k-1}(I R F)\right]^{-1} A^{T}\right\}^{-1}=J_{k}(I R F)
\end{gathered}
$$

In formula (8), IRF is the CRLB lower bound in information reduction factor method, and the calculation is small. Thus the lower bound of residual effectiveness under incomplete measurement can be get :

$$
\sigma_{(i, i) \min }=J_{v(i, i), k}^{l}
$$

Of course, as an important measurement tool of filter estimate, CRLB has been applied into the fusion system optimization design and sensor online management ${ }^{[6]}$. Therefore, as the research on lower bound of sensor measurement information effectiveness under incomplete measurement 
condition, CRLB has significant theoretical meaning and practical application value.

\section{Detection criterion of upper and lower bounds based on residual effectiveness}

This article makes residual normalized due to that residual a multi-way matrix, and detects value $d_{k}$ of incomplete measurement information according to the detected index which is the trace $\xi_{k}=\operatorname{tr}[\psi(k+1 \mid k)]$ of $\psi(k+1 \mid k)$. So the following conclusions can be get:

1) When $\xi_{k} \leq r_{2}, \hat{d}_{k}=1$, effectiveness of the measurement residual is the highest at this time and the value of it is 1 ;

2) When $\xi_{k}>r_{1}, \hat{d}_{k}=0$, effectiveness of the measurement residual is 0 , incomplete measurement appears, which makes the measure value is useless.

3) When $r_{2}<\xi_{k} \leq r_{1}$, value that $\hat{d}_{k}=0, \hat{d}_{k}=1$ exists at a certain probability. According to fuzzy theory, applying trapezoid fuzzy function to make value between the upper bound and lower bound fuzzy, thus the effective degree of measurement residual can be get:

$$
\mu\left(\xi_{k}\right)=\left\{\begin{array}{lr}
1, & \xi_{k} \leq r_{2} \\
-\frac{\xi_{k}-r_{1}}{r_{1}-r_{2}}, & r_{2}<\xi_{k} \leq r_{1} \\
0, & \xi_{k}>r_{1}
\end{array}\right\}
$$

Assuming that effective data is $M$, then $P\left(M \mid \xi_{k}\right)=\mu$, the prior probability of which is $P(M)=\lambda$, and the posterior probability can be get on a further stair on the basis of Bayes formula in signal detection theory:

$$
P\left(M \mid \xi_{k}\right)=\frac{P(M) P\left(\xi_{k} \mid M\right)}{P\left(\xi_{k}\right)}=\frac{P(M) P\left(\xi_{k} \mid M\right)}{P(M) P\left(\xi_{k} \mid M\right)+P(\bar{M}) P\left(\xi_{k} \mid \bar{M}\right)}=\frac{\lambda \cdot \mu}{\lambda \cdot \mu+(1-\lambda) \cdot(1-\mu)}
$$

In formula (11), $\bar{M}$ is ineffective measurement information. Assuming that the threshold based on effective data is $r_{3}$, the following formula can be get:

$$
\hat{d}_{k}=1, \quad P\left(M \mid \xi_{k}\right) \geq r_{3} ; \hat{d}_{k}=0, \quad P\left(M \mid \xi_{k}\right)<r_{3}
$$

\section{Simulation verification}

For example: the target is making CV model move in $6000 \mathrm{~m}$ altitude high during $0 \mathrm{~s}$ to $600 \mathrm{~s}$, apply different norm to judge in random data drop in the time quantum. Suppose this moment sensor's detection probability $\lambda=0.65$, we can get the target's real move track and sensor measure dot trace as figure 1 .

From figure 1 we can find that some moment the measure value only from noise except normal measure value, and part measure value is severe deviate target real value, if directly filter the effectiveness of filter must be influenced.

Corresponding with figure1, figure 3 offers different moment sensor real measure value. In which $d_{k}=1$ represent $\mathrm{K}$ moment sensor is measuring, $d_{k}=0$ indicate the time have no target's measure value, arising incomplete measure.

The figure 3 offer the residual detection result of application effectiveness upper and lower 
bounds. Comparing the figure 2 and figure 3 we can see the norm can detect $d_{k}$ then get the moment real value of $d_{k}$, because this time sensor's detection probability rather low, when $k=6,12,26,30,35,43,48,58$ arising detecting error.

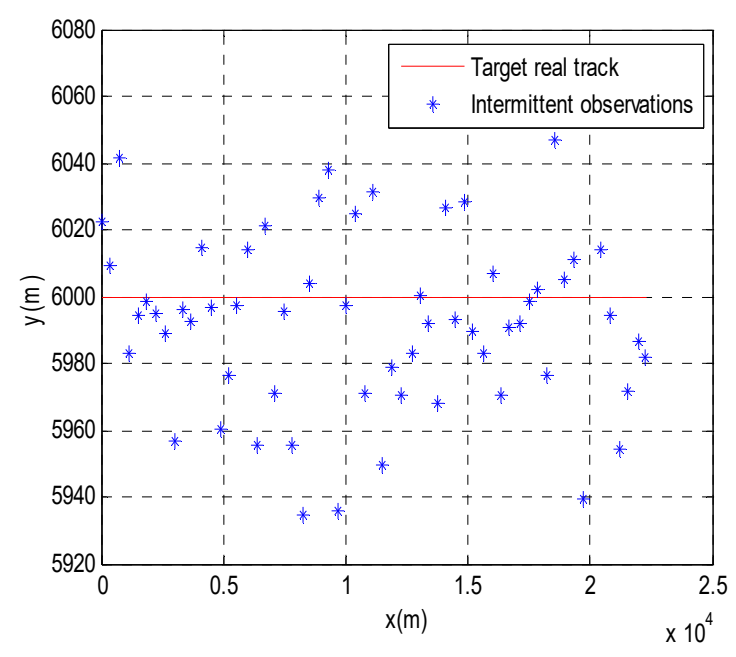

Figure 1 Sensor measurement point trace

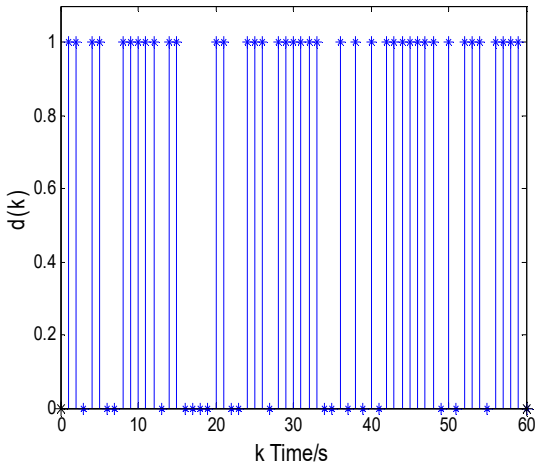

Figure 2 True value of $d_{k}$

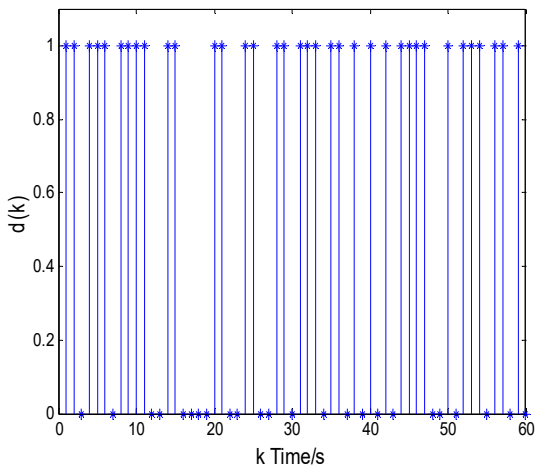

Figure 3 Detection value of $d_{k}$

Table 1 contrast the detection correct probability under different criterion. From the table former 4 norms all set single all according to the size of residual in filter processing. UBL based on the effectiveness of residual shows higher detection performance under different detection probability.

Table 1 Contrast of detection correct probability under different criterion

\begin{tabular}{|c|c|c|c|}
\hline \multirow{2}{*}{ Performance } & \multicolumn{3}{|c|}{ Detection accuracy } \\
\cline { 2 - 4 } & Detection probability 0.9 & Detection probability 0.8 & Detection probability0.7 \\
\hline $3 \sigma$ & $81.3 \%$ & $72.8 \%$ & $63.3 \%$ \\
\hline Nair's & $85.3 \%$ & $77.6 \%$ & $67.4 \%$ \\
\hline Grubbs & $82.4 \%$ & $75.3 \%$ & $68.8 \%$ \\
\hline Dixon & $90.2 \%$ & $81.6 \%$ & $72.1 \%$ \\
\hline UBL & $97.3 \%$ & $90.0 \%$ & $82.6 \%$ \\
\hline
\end{tabular}

\section{Conclusions}

This paper points at analyzing single sensor measure residual information effectiveness and combines outliers' judge rule, proposing basing upon effectiveness limitation residual detection norm, and designing basing upon residual information revise incomplete measure trace filter. Then 
make a simulation experiment for confirming. The result indicate higher detect performance in different detection probability basing upon residual effectiveness up and low limit norm.

\section{Acknowledgements}

This research was funded by the Science and Technology on Avionics Integration Laboratory \& Aeronautical Science Fund (20145596025); This research was funded by the Science and Technology on Avionics Integration Laboratory \& Aeronautical Science Fund (20155596024).

\section{References}

[1] J. Manyika, H. Durrant-Whyte. Data fusion and sensor management: a decentralized information-theoretic approach.

[2] D. A. Castañón, Approximate dynamic programming for sensor management. in Proc. of IEEE Conf. Decision and Control, vol. 2, 1997, pp. 1202-1207.

[3] Nahi N E. Optimal recursive estimation with uncertain observation[J]. IEEE Transactions on Information Theory, 1969, 15(4): 457-462.

[4] Hong-fa Ke, Yong-guang Chen, Jin-liang Wu. Uncertainty information processing of effectiveness evaluation for electronic warfare equipment[J]. Military Operations Research and Systems Engineering, 2007. 4: 3(1):36-40.

[5] Jun Tong, Gan-lin Shan. Multi-sensor tracking resource coordinated allocation based on Cramér-Rao low bound[J]. Journal of Astronautics, 2012(9), 33(9):1314-1321.

[6] P. Shi, M. Mahmoud, S. K. Nguang, A. Ismail. Robust filtering for jumping systems with mode-dependent delays[J]. Signal Processing, 2006, Vol. 86, No. 1, pp. 140-152. 